\title{
Spin relaxation in a Rashba semiconductor in an electric field
}

\author{
O.Bleibaun $*$ \\ Institut für Theoretische Physik, Otto-von-Guericke Universität Magdeburg, PF4120, 39016 Magdeburg, Germany
}

\begin{abstract}
The impact of an external electric field on the spin relaxation in a disordered, two-dimensional electron system is studied within the framework of a field-theoretical formulation. Generalized Bloch-equations for the diffusion and the decay of an initial magnetization are obtained. The equations are applied to the investigation of spin relaxation processes in an electric field.
\end{abstract}

PACS numbers: 72.10.-d, 73.50.-h

\section{INTRODUCTION}

In recent years much attention has been paid to the emerging field of spintronics in semiconductor physics. Much of the interest in this field is stimulated by the spin-field-effect transistor proposed by Datta and Das 1 . In this proposal the Rashba spin-orbit interaction is used to manipulate the charge transfer through a transistor like device by controlling the electron spin. The Rashba interaction, which is the conventional spin-orbit interaction with a constant electric field, affects the electron spin like a momentum dependent magnetic field.

In practice the realization of the proposal by Datta and Das has proven to be a challenging task. In particular the injection of spins into non-magnetic semiconductors has turned out to be demanding, although successful injection has been reported by several authors ${ }^{2.3 .4 .5 .6}$. Despite this fact the importance of the Rashba interaction for the concept of the spin-field effect transistor has motivated further studies on its impact on charge and spin transport properties. Particular attention has been paid, e.g., to investigations of spin-accumulations ${ }^{7}$ and to the spin-galvanic effect ${ }^{8}$, to an intrinsic spin-Hall effect ${ }^{9}$, and to the weak localization-antilocalization transition in the conductivity 10.11.12.13.14.

Since in the Rashba model the electron wave-vector is coupled to the spin the spin is quickly randomized by scattering events. Therefore, the ballistic transport regime is the most important regime for the spin-field effect transistor proposed by Datta and Das. Accordingly, most of the investigations focus on the situation in which the disorder energy is small compared to the Rashba level splitting. Recently, however, a generalized spin-field effect transistor has been proposed ${ }^{15}$ which is also expected to work in the diffusive regime, even if the Rashba level splitting is small compared to the characteristic disorder energy. This raises the question which impact of the Rashba interaction on the charge and spin transport properties can be expected in this limit.

The aim of the paper is to provide a theory for the spinrelaxation in a a two-dimensional electron gas in the diffusive regime, in the limit that the characteristic disorder

*Electronic address: olaf.bleibaum@physik.uni-magdeburg.de energy is large compared to the Rashba level splitting on a time scale, in which inelastic processes can be ignored. To this end we generalize the non-nonlinear $\sigma$-model to include also the impact of the Rashba interaction and an in-plane electric field. Using the generalized $\sigma$-model we derive generalized Bloch-equation and use these equations for the investigation of an initial magnetization.

\section{BASIC EQUATIONS}

We consider charge carriers in a two-dimensional plane. The motion of the charge carriers is described by the Hamilton operator

$$
H=\frac{\hat{\boldsymbol{p}}^{2}}{2 m}+\boldsymbol{F} \cdot \boldsymbol{x}+\boldsymbol{\sigma} \cdot(\boldsymbol{N} \times \hat{\boldsymbol{p}})+V(\boldsymbol{x}) .
$$

Here $\hat{\boldsymbol{p}}$ is the momentum operator, $m$ is the effective mass, $\boldsymbol{F}$ is a constant field in the two-dimensional plane, $\boldsymbol{N}$ is a constant vector perpendicular to the plane, and $V(\boldsymbol{x})$ is a random potential. The components of the vector $\boldsymbol{\sigma}=\sigma_{x} \boldsymbol{e}_{x}+\sigma_{y} \boldsymbol{e}_{y}+\sigma_{z} \boldsymbol{e}_{z}$ are the Pauli matrices. $\boldsymbol{e}_{x}$ and $\boldsymbol{e}_{y}$ are the unit vectors in the two-dimensional plane and $\boldsymbol{e}_{z}$ is a unit vector transverse to the plane. Accordingly, $\boldsymbol{N}=N_{z} \boldsymbol{e}_{z}$ and $\boldsymbol{F}=F \boldsymbol{e}_{x}$. The random potential is characterized by a Gaussian distribution function with zero mean and second moment

$$
\left\langle V(\boldsymbol{x}) V\left(\boldsymbol{x}^{\prime}\right)\right\rangle_{d i s}=\frac{1}{\pi \nu \tau} \delta\left(\boldsymbol{x}-\boldsymbol{x}^{\prime}\right) .
$$

Here $\langle\ldots\rangle_{\text {dis }}$ denotes the configuration average, $\nu$ is the density of states per spin at the Fermi-level for $N_{z}=0$, and $\tau$ is the single-particle scattering time.

In our investigation we focus on configuration averages of retarded and advanced Green functions. These functions are solutions to the differential equation

$$
(( \pm i \omega+E) \mathbf{1}-H) G^{R / A}\left(\boldsymbol{x}, \boldsymbol{x}^{\prime} \mid E, \omega\right)=\mathbf{1} \delta\left(\boldsymbol{x}-\boldsymbol{x}^{\prime}\right) .
$$

In this equation $G^{R / A}$ is a $2 \times 2$ matrix in spin-space, $\mathbf{1}$ is the $2 \times 2$ unit matrix and $E$ and $\omega$ are fixed energies. As a boundary condition we require that the Green functions vanish at infinity. In this case the configuration averaged Green functions are invariant against translations by a fixed vector $\mathbf{a}$ if the energy $E$ is also shifted by $\boldsymbol{F} \cdot \boldsymbol{a}$. 
Accordingly, they satisfy the relationship

$$
\begin{aligned}
\left\langle G^{R / A}\right\rangle_{\text {dis }} \quad( & \left.\boldsymbol{x}+\boldsymbol{a}, \boldsymbol{x}^{\prime}+\boldsymbol{a} \mid E, \omega\right) \\
& =\left\langle G^{R / A}\right\rangle_{\text {dis }}\left(\boldsymbol{x}, \boldsymbol{x}^{\prime} \mid E-\boldsymbol{F} \cdot \boldsymbol{a}, \omega\right) .
\end{aligned}
$$

To calculate the configuration averages of the Green functions we use the field theoretical formulation of the Refs. [16 17 18]. In this formulation the replica trick is used to derive an effective action for the calculation of the configuration averaged Green functions. Following the standard derivation we find that for the problem at hand the partition function takes the form

$$
Z=\int D Q \exp \left(A_{Q}\right)
$$

where

$$
A_{Q}=-\frac{\pi \nu \hbar}{8 \tau} \operatorname{tr} Q^{2}+\frac{1}{2} \operatorname{tr} \ln G_{Q}^{-1}
$$

Here $Q$ is a hermitian $\left(2 n_{r}+4\right) \times\left(2 n_{r}+4\right)$ matrix field $\left(n_{r}\right.$-number of replica copies +2 components particlehole space +2 components spin indices) with components $Q_{\alpha m \alpha^{\prime} n}^{i j}(\boldsymbol{x})\left(\alpha, \alpha^{\prime}\right.$ - replica indices, $i, j$-particle-hole indices, $m, n$-spin indices). It satisfies the relationship $Q C=C Q^{T}$, where $Q^{T}$ is the transposed matrix and

$$
C=\left(\begin{array}{cc}
0 & i \sigma_{y} \\
i \sigma_{y} & 0
\end{array}\right)
$$

The tr in Eq.(2.6) contains both the summation over the discrete indices and the integration over space. Furthermore,

$$
\begin{aligned}
G_{Q}^{-1}{ }_{\alpha m \alpha^{\prime} n}^{i j}\left(\boldsymbol{x}, \boldsymbol{x}^{\prime}\right)= & {\left[\left\{\left(i \omega_{\alpha}+E\right) \delta_{m n}-H_{m n}\right\} \delta_{\alpha \alpha^{\prime}} \delta_{i j}\right.} \\
& \left.+i \frac{\hbar}{2 \tau} Q_{\alpha m \alpha^{\prime} n}^{i j}(\boldsymbol{x})\right] \delta\left(\boldsymbol{x}-\boldsymbol{x}^{\prime}\right),(2.8
\end{aligned}
$$

where $\omega_{\alpha}=\omega \Lambda_{\alpha}, \Lambda_{\alpha}=1$ for $\alpha>0$ and $\Lambda_{\alpha}=-1$ for $\alpha \leq 0$. The index $\alpha$ can take on values between $-\left(n_{r}-1\right)$ and $n_{r}$. It is understood that the limit $n_{r} \rightarrow 0$ is taken at the end of the calculation.

\section{THE SADDLE-POINT GREEN FUNCTIONS}

To calculate the configuration-averaged Green function in saddle-point approximation we now look on the saddlepoint solution of the effective action $A_{Q}$, defined by

$$
\left.\frac{\delta A_{Q}}{\delta Q}\right|_{Q=Q_{S P}}=0 .
$$

The saddle-point values of $Q$ and $G_{Q}$ are diagonal with respect to the replica indices, as are the exact expection values. Accordingly, $Q_{S P}{ }_{\alpha m \alpha^{\prime} n}^{i j}=Q_{\alpha m n}{ }^{i j} \delta_{\alpha \alpha^{\prime}}$ and

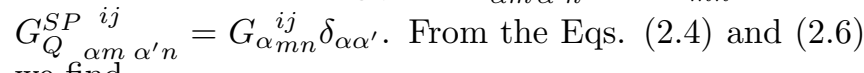
we find

Here $\mu_{x}=E-\boldsymbol{F} \cdot \boldsymbol{x}$.

$$
\begin{aligned}
Q_{\alpha m n}^{i j}(\mathbf{x}) & =\frac{i}{\pi \nu} G_{\alpha m n}^{i j}(0,0 \mid E-\boldsymbol{F} \cdot \boldsymbol{x}, \omega) \\
& \equiv \tilde{Q}_{\alpha}^{i j}\left(\mu_{x}\right) .
\end{aligned}
$$

To find an equation for the Green function we use the gradient expansion ${ }^{19}$. To this end we first introduce Wigner coordinates, according to $\boldsymbol{R}=\left(\boldsymbol{x}+\boldsymbol{x}^{\prime}\right) / 2$ and $\boldsymbol{r}=\boldsymbol{x}-\boldsymbol{x}^{\prime}$, and perform a Fourier transformation with respect to the relative coordinates. The Green function in the new coordinates $\tilde{G}$ is related to the old Green function by the relationship

$$
\begin{aligned}
G_{\alpha} \underset{m n}{i j}( & \boldsymbol{R}+\boldsymbol{r} / 2, \boldsymbol{R}-\boldsymbol{r} / 2 \mid E, \omega) \\
= & \int \frac{d \boldsymbol{k}}{(2 \pi)^{2}} e^{i \boldsymbol{k} \cdot \boldsymbol{r}} \tilde{G}_{\alpha \stackrel{m}{i j}(\boldsymbol{R}, \boldsymbol{k} \mid E, \omega) .}
\end{aligned}
$$

In calculating $\tilde{G}$ we restrict the consideration to the lowest order in the gradient expansion. In this approximation the equation for the Green function takes the simple form

$$
\sum_{j}\left(\left[\left(i \omega_{\alpha}+\mu_{R}-\epsilon_{k}\right) \mathbf{1}-\hbar \boldsymbol{\sigma} \cdot(\boldsymbol{N} \times \boldsymbol{k})\right] \delta_{i j}+i \frac{\hbar}{2 \tau} \tilde{Q}_{\alpha}^{i j}\left(\mu_{R}\right)\right) \tilde{G}_{\alpha}^{j k}(\boldsymbol{R}, \boldsymbol{k} \mid E, \omega)=\mathbf{1} \delta_{i k}
$$

Here all objects are considered as $2 \times 2$ matrices in spin space, $\epsilon_{k}=\hbar^{2} k^{2} / 2 m$ and $\mu_{R}=\left.\mu_{x}\right|_{\boldsymbol{x}=\boldsymbol{R}}$.

In order to solve the Eqs.(3.2)-(3.4) self-consistently we restrict the consideration to the classical accessible region $\mu_{R}>0$. Furthermore, we consider only the dirty limit, in which both the disorder energy $\hbar / 2 \tau$ and $\mu_{R}$ are large compared to the Rashba level splitting $\left|N_{z}\right| \sqrt{2 m \mu_{R}}$ and assume that $\mu_{R}$ is large compared to $\hbar / 2 \tau$. In this case we find that the self-consistent solution to the equations (3.2) and (3.4) takes the form

$$
\tilde{Q}_{\alpha} \stackrel{i j}{m n}\left(\mu_{R}\right)=\delta_{m n} \delta_{i j} \operatorname{sgn} \omega_{\alpha}
$$




$$
\tilde{G}_{\alpha} \underset{m n}{i j}(\boldsymbol{R}, \boldsymbol{k} \mid E, \omega)=g_{\alpha m n}\left(\boldsymbol{k} \mid \mu_{R}, \omega\right) \delta_{i j},
$$

$$
\begin{gathered}
g_{\alpha}\left(\boldsymbol{k} \mid \mu_{R}, \omega\right)=\frac{1}{M_{\alpha}\left(k \mid \mu_{R}, \omega\right)}\left(\begin{array}{cc}
i \omega_{\alpha}+\mu_{R}-\epsilon_{k}+i \frac{\hbar}{2 \tau} \operatorname{sgn} \omega_{\alpha} & -i N_{z} \hbar k_{-} \\
i N_{z} \hbar k_{+} & i \omega_{\alpha}+\mu_{R}-\epsilon_{k}+i \frac{\hbar}{2 \tau} \operatorname{sgn} \omega_{\alpha}
\end{array}\right), \\
M_{\alpha}\left(\boldsymbol{k} \mid \mu_{R}, \omega\right)=\left(i\left(\omega_{\alpha}+\frac{\hbar}{2 \tau} \operatorname{sgn} \omega_{\alpha}\right)+\mu_{R}-E_{+}(k)\right)\left(i\left(\omega_{\alpha}+\frac{\hbar}{2 \tau} \operatorname{sgn} \omega_{\alpha}\right)+\mu_{R}-E_{-}(k)\right),
\end{gathered}
$$

where $k_{ \pm}=k_{x} \pm i k_{y}$ and $E_{ \pm}=\epsilon_{k} \pm \hbar\left|N_{z}\right| k$. In writing down the saddle-point field (3.5) we have ignored its imaginary part which only leads to an $\alpha$-independent shift of $\mu_{R}$. We would like to note that the simple structure of the saddle-point field is a consequence of the fact that the density of states for $N_{z}=0$ is constant. In an energy dependent density of states the saddle-point would pick up an energy and field dependent contribution from the density of states, which would modify Eq.(3.5).

\section{THE NON-LINEAR $\sigma$-MODEL}

The fluctuations around the saddle-point solution are characterized by massive and soft modes. The latter describe the diffusion and spin relaxation properties we are interested in. In the field-theoretical formulation the existence of these modes is a consequence of a continuous symmetry of the effective action (2.6) which exists in the limit $\omega, N_{z} \rightarrow 0$. In this limit the effective action is invariant against similarity transformations with hermitian matrices $B$, which satisfy the relationship

$$
B^{T} C B=C .
$$

Such matrices are generated by hermitian generators $W$ $(B=\exp (i W))$, which have the structure

$$
\begin{aligned}
W_{\alpha \alpha^{\prime}}= & \sum_{\lambda=0}^{2}\left[i A_{\alpha \alpha^{\prime}{ }_{0}{ }_{0}} \sigma_{\lambda} \otimes \sigma_{0}+\sum_{i=1}^{3} S_{\alpha \alpha^{\prime}{ }_{i}{ }_{i}} \sigma_{\lambda} \otimes \sigma_{i}\right] \\
& +S_{\alpha \alpha^{\prime}{ }_{0}{ }^{3} \sigma_{3} \otimes \sigma_{0}+i A_{\alpha \alpha^{\prime} i}{ }^{3} \sigma_{3} \otimes \sigma_{i}}
\end{aligned}
$$

Here $S_{\alpha \alpha^{\prime}{ }_{\kappa}{ }_{k}}\left(A_{\alpha \alpha^{\prime}}{ }^{\lambda}\right)$ are real matrices symmetric (antisymmetric) in $\alpha$ and $\alpha^{\prime}, \sigma_{0}=\mathbf{1}$, and $i=1,2,3$ corresponds to $x, y$ and $z$, respectively. The components of the matrices $W_{\alpha \alpha^{\prime}}$, which are associated with the upper index of the matrices $S_{\alpha \alpha^{\prime}{ }_{\kappa}{ }_{k}}$ and $A_{\alpha \alpha^{\prime}{ }_{\kappa}{ }_{k}}$ (that is with $\lambda$ ), correspond to the particle-hole indices. The lower indices characterize the spin.

In order to derive an effective action for the soft modes we follow the standard derivation for the nonlinear sigma-mode1 16.17 .18 . The complications originating from the presence of the electric field we treat as in Ref. [20]. Doing so, we find that the effective action for the diffusion and spin relaxation modes takes the form

$$
A_{\sigma}=\frac{\pi \nu \omega}{2} \int d \boldsymbol{x} \theta\left(\mu_{x}\right) \operatorname{tr}(\Lambda \hat{Q}(\boldsymbol{x}))-\frac{\pi \nu \hbar}{8} \int d \boldsymbol{x} \theta\left(\mu_{x}\right) D\left(\mu_{x}\right) \operatorname{tr}(\boldsymbol{D} \hat{Q}(\boldsymbol{x}) \cdot \boldsymbol{D} \hat{Q}(\boldsymbol{x})) .
$$

Here $\hat{Q}(\boldsymbol{x})$ is the soft part of the matrix $Q(\boldsymbol{x})$ generated from the saddle-point field $Q_{S P}(\boldsymbol{x})$ by the local similarity transformation $\hat{Q}(\boldsymbol{x})=B(\boldsymbol{x}) Q_{S P}(\boldsymbol{x}) B^{-1}(\boldsymbol{x})$,

$$
D\left(\mu_{x}\right)=\frac{\tau \mu_{x}}{m},
$$

and

$$
\boldsymbol{D} \hat{Q}(\boldsymbol{x})=\nabla \hat{Q}(\boldsymbol{x})-i \frac{m}{\hbar}[\boldsymbol{\sigma} \times \boldsymbol{N}, \hat{Q}(\boldsymbol{x})] .
$$

The bracket $[\ldots, \ldots]$ symbolizes the commutator and the step function $\theta\left(\mu_{x}\right)$ restricts the range of integration to the classical accessible region. The matrix $\boldsymbol{\sigma} \times \boldsymbol{N}$ in Eq. (4.5) is a matrix which only acts on the spin-indices but not on the replica and the particle-hole indices.

In order to investigate the non-linear $\sigma$-model further 
we write the matrix $\hat{Q}$ in the form

$$
\hat{Q}=\left(\begin{array}{cc}
\sqrt{1-q_{12} q_{21}} & q_{12} \\
q_{21} & -\sqrt{1-q_{21} q_{12}}
\end{array}\right),
$$

where

$$
q_{12} \equiv q_{\alpha \alpha^{\prime}}{ }_{\lambda}^{\kappa} \sigma_{\kappa} \otimes \sigma_{\lambda},
$$

$\alpha>0, \alpha^{\prime} \leq 0$ and $q_{21}=q_{12}^{+}$, where + symbolizes hermitian conjugation, and expand the action (4.3) with respect to powers of $q$ up to fourth order in $q$. Doing so, we use the fact that the matrices $q_{\alpha \alpha^{\prime}}{ }_{\kappa}^{\lambda}$ are antisymmetric with respect to $\alpha$ and $\alpha^{\prime}$ for $\lambda=0,1,2$ and $\kappa=0$ and for $\lambda=3$ and $\kappa=1,2,3$ and symmetric with respect to $\alpha$ and $\alpha^{\prime}$ for $\lambda=3$ and $\kappa=0$ and for $\lambda=0,1,2$ and $\kappa=1,2,3$. The quadratic terms in the expansion yield the Gaussian propagator, the physics of which agrees with the ladder-approximation. The quartic terms in the expansion yield the weak-localization corrections to the transport coefficients.

\section{A. Diffusion and relaxation in the Gaussian approximation}

In the Gaussian approximation the effective action takes the form

$$
\begin{aligned}
A_{\sigma}^{(2)}=-\pi \nu & \hbar \sum_{\substack{\alpha>0 \\
\alpha^{\prime} \leq 0}} \sum_{\lambda, \kappa, \delta} \int d \boldsymbol{x} \theta\left(\mu_{x}\right) \\
& \times q_{\alpha \alpha^{\prime}}{ }_{\lambda}^{\kappa}(\boldsymbol{x}) \Gamma_{\lambda \delta}(\boldsymbol{x} \mid E, s) \eta_{\delta}^{\kappa} q_{\alpha \alpha^{\prime}{ }^{\prime} \delta}(\boldsymbol{x}),
\end{aligned}
$$

where $\eta_{\lambda}^{\kappa}=1$ for $\lambda=0,1,2$ and $\kappa=1,2,3$ and for $\lambda=3$ and $\kappa=0$ and $\eta_{\lambda}^{\kappa}=-1$ else. The operator $\Gamma_{\lambda \delta}(\boldsymbol{x} \mid E, s)$, the non-vanishing components of which are

$$
\Gamma_{00}(\boldsymbol{x} \mid E, s)=s-\left(\boldsymbol{\nabla}, D\left(\mu_{x}\right) \boldsymbol{\nabla}\right)
$$

and

$$
\begin{aligned}
\Gamma_{i k}(\boldsymbol{x} \mid E, s) & =\left(s+\Omega_{i}\left(\mu_{x}\right)\left(1+\delta_{i 3}\right)-\left(\boldsymbol{\nabla}, D_{i}\left(\mu_{x}\right) \nabla\right)\right) \delta_{i k} \\
& +\frac{N_{z}}{2}\left(\delta_{k 3}\left\{\omega_{s}\left(\mu_{x}\right), \nabla_{i}\right\}-\left\{\omega_{s}\left(\mu_{x}\right), \nabla_{i}\right\} \delta_{i 3}\right),
\end{aligned}
$$

is related to the generalized diffusion propagator $P_{\lambda \kappa}\left(\boldsymbol{x}, \boldsymbol{x}^{\prime} \mid E, s\right)$ by the relationship

$$
\sum_{\lambda} \Gamma_{\kappa \lambda}(\boldsymbol{x} \mid E, s) P_{\lambda \delta}\left(\boldsymbol{x}, \boldsymbol{x}^{\prime} \mid E, s\right)=\delta_{\kappa \delta} \delta\left(\boldsymbol{x}-\boldsymbol{x}^{\prime}\right) .
$$

Here the bracket $\{. ., .$.$\} symbolizes the anti-commutator,$ $\hbar s=\omega / 2$ and the indices $i$ and $k$ can take on the values $1,2,3$, which correspond to $x, y$, and $z$, respectively. The transport coefficients are given by

$$
\Omega_{i}\left(\mu_{x}\right)=\frac{4 N_{z}^{2} m^{2}}{\hbar^{2}} D\left(\mu_{x}\right)
$$

$$
\omega_{s}\left(\mu_{x}\right)=\frac{4 m}{\hbar} D\left(\mu_{x}\right)
$$

and $D_{i}\left(\mu_{x}\right)=D\left(\mu_{x}\right)$.

According to the Eqs. (4.9), (4.10) and (4.11) the equation for the particle transport decouples from the equations for the spin transport. The particle transport is described by the propagator $P_{00}$. Its equation of motion contains besides ordinary particle diffusion also the particle packet drift and the heating of the charge carriers. Its physics is detailed in Ref.[20]. The spatial components of the diffusion propagator, $P_{i k}$ for $i, k=1,2,3$, describe spin diffusion and relaxation. In addition to the diffusion, drift and heating processes present in the particle number diffusion equation, these equations also contain the spin relaxation and an electric-field dependent rotation of the spin-direction, which is induced by the Rashba-interaction. We would like to note that the same equations can also be obtained from the ladder approximation.

\section{B. Quantum corrections}

In order to calculate the quantum corrections to the transport coefficients we also take into account the quartic terms in the expansion of $A_{\sigma}$ with respect to $q$ and investigate the corresponding functional integral in oneloop approximation. Doing so, we restrict the consideration to the linear response regime. In this case we can ignore the impact of the electric field on the transport coefficients and thus set the external electric field $\boldsymbol{F}$ equal to zero in all loops. For charge transport the range of validity of this approximation has been discussed in Ref. 20]. There it has been shown that the impact of the field is negligible, as long as the spread of the particle packet is small compared to the distance of its center from the turning point. The time needed by a particle packet, which was a delta-pulse centered at $\boldsymbol{x}=\mathbf{0}$ at time $t=0$ and in which all particles have the same energy $E$, to reach a width of the order of $E / F$ is for a typical experiment larger than $10^{-4} \mathrm{~s}$, as shown in Ref. [20]. Given that the typical lifetime of an initial magnetization is in the nanosecond range it seems to be reasonable to restrict the consideration to this limit.

To one-loop order the particle number diffusion coefficient $D(E)$ in Eq. (4.9) is replaced by

$$
\begin{aligned}
D^{(1)}(E, s)=D(E)\left(1-\frac{1}{2 \pi \nu \hbar}(\right. & P_{33}(E, s)+2 P_{11}(E, s) \\
& \left.\left.-P_{00}(E, s)\right)\right),
\end{aligned}
$$

where

$$
\begin{gathered}
P_{00}(E, s)=\frac{1}{4 \pi D(E)} \ln \frac{D(E) \tilde{\Lambda}^{2}}{s}, \\
P_{11}(E, s)=\frac{1}{4 \pi D(E)} \ln \frac{D(E) \tilde{\Lambda}^{2}}{s+\Omega(E)},
\end{gathered}
$$


and

$$
P_{33}(E, s)=\frac{1}{4 \pi D(E)} \ln \frac{D(E) \tilde{\Lambda}^{2}}{\sqrt{s^{2}+2 \Omega^{2}(E)+3 s \Omega(E)}} .
$$

Here $\tilde{\Lambda}$ is the ultraviolet cutoff. These corrections have the same structure as the conventional anti-weak localization corrections to the conductivity ${ }^{21}$. In a current relaxation experiment the frequency $s$ is effectively replaced by $1 / \tau_{\phi}(T)$, where $\tau_{\phi}(T)$ is the inelastic phase breaking time which increases with decreasing temperature. Accordingly, the particle number diffusion coefficient has a minimum as a function of the temperature at $T=T_{\Omega}$, where $\Omega(E) \approx 1 / \tau_{\phi}\left(T_{\Omega}\right)$. Therefore, the diffusion coefficient increases with decreasing temperature if $T<T_{\Omega}$.

The opposite turns out to be true for the spin-transport coefficients. To one-loop order the spin diffusion coefficients $D_{x}(E)$ and $D_{y}(E)$ are replaced by

$$
D_{x / y}^{(1)}(E, s)=D(E)\left(1-\frac{1}{2 \pi \nu \hbar}\left(P_{00}(E, s)+P_{33}(E, s)\right)\right),
$$

$D_{z}(E)$ by

$$
\begin{aligned}
D_{z}^{(1)}(E, s)=D(E)\left(1-\frac{1}{2 \pi \nu \hbar}(\right. & P_{00}(E, s)+2 P_{11}(E, s) \\
& \left.\left.-P_{33}(E, s)\right)\right),
\end{aligned}
$$

the spin relaxation frequencies $\Omega_{i}(E)$ by

$$
\Omega_{x / y}(E, s)=\Omega(E) \frac{D_{z}^{(1)}(E, s)}{D(E)}
$$

and

$$
\Omega_{z}^{(1)}(E, s)=\Omega(E) \frac{D_{x}^{(1)}(E, s)}{D(E)},
$$

and the rotation frequency $\omega_{s}(E)$ by

$$
\omega_{s}^{(1)}(E, s)=\omega_{s}(E)\left(1-\frac{1}{2 \pi \nu \hbar}\left(P_{00}(E, s)+P_{11}(E, s)\right)\right) .
$$

In these equations $s$ has also to be replaced by $1 / \tau_{\phi}(T)$ if $s$ is smaller than $1 / \tau_{\phi}(T)$. In contrast to the particlenumber diffusion coefficient, however, the spin-transport coefficients have no minimum as a function of the temperature. In the range of applicability of the Eqs. (4.18)(4.22) the magnitude of the spin transport coefficients keeps decreasing with decreasing temperature even if $T<T_{\Omega}$. In contrast to the corrections to the particlenumber diffusion coefficient, which are anti-localizing at low temperatures, the corrections to the spin transport coefficients always show a tendency to localization.

At this point we would also like to mention that the quantum corrections render the spin-transport coefficients weakly anisotropic. This anisotropy results from the fact that $P_{11}(E, s) \neq P_{33}(E, s)$. However, since the difference between these quantities is of the order of $1 / 4 \pi \nu \hbar D(E)$ this difference is small compared to the magnitude of the quantum corrections themselves, which are larger than the former by a factor of the order of $\ln \left(D(E) \tilde{\Lambda}^{2} /(s+\Omega(E))\right)$. Therefore, we ignore the anisotropy in the following and replace $P_{33}(E, s)$ by $P_{11}(E, s)$ everywhere. In this case $\Omega_{x}^{(1)}(E, s)=$ $\Omega_{z}^{(1)}(E, s)=\Omega^{(1)}(E, s)$ and $D_{x}^{(1)}(E, s)=D_{z}^{(1)}(E, s)=$ $D_{s}^{(1)}(E, s)$

\section{APPLICATION TO RELAXATION PHENOMENA}

The evolution of an initial spin packet is described by the Bloch equations. Phenomenology tells us that the Bloch equations have the structure

$$
s \boldsymbol{S}(s)+\boldsymbol{\Omega}(s) \cdot \boldsymbol{S}(s)+\boldsymbol{R}(s) \times \boldsymbol{S}(s)+\operatorname{div} \boldsymbol{J}(s)=\boldsymbol{S}_{0} .
$$

Here $\boldsymbol{S}(s)$ is the Laplace transform of the spin density with respect to time, $s$ is the corresponding Laplace frequency, $\boldsymbol{S}_{0}$ is the initial condition, $\boldsymbol{\Omega}(s)$ is a second rank tensor, $\boldsymbol{R}(s)$ is a vector, and $\boldsymbol{J}(s)$ is the spincurrent tensor. For the components we use the notation $(\boldsymbol{\Omega} \cdot \boldsymbol{S})_{i}=\sum_{k} \Omega_{i k} S_{k}$ and $(\operatorname{div} \boldsymbol{J})_{i}=\sum_{k} \nabla_{k} J_{k i}$. The quantities $\boldsymbol{S}(s), \boldsymbol{S}_{0}$ and $\boldsymbol{J}(s)$ depend also on $\boldsymbol{x}$ and on the total energy $E$, but this dependence has been suppressed in Eq. (5.1) to simplify the notation. Due to the generalized translation invariance (2.4) the transport coefficients $\boldsymbol{\Omega}$ and $\boldsymbol{R}$ do not depend on the quantities $\boldsymbol{x}$ and $E$ separately, but only on the kinetic energy $\mu_{x}$. In Eq. (5.1) this dependence has also been suppressed to simplify the notation.

From the physical point of view the tensor $\boldsymbol{\Omega}(s)$ describes the decay of the initial magnetization and $\boldsymbol{R}(s)$ describes the rotation of the initial magnetization. The disorder renders both quantities dispersive. Therefore, both $\boldsymbol{\Omega}(s)$ and $\boldsymbol{R}(s)$ depend on $s$. This dependence should be observable in optical experiments where it should reflect itself in deviations from the purely exponential decay.

The equations (4.10) and (4.11) can be cast into the form (5.1). Doing so, it has to be taken into account that the bare transport coefficients are renormalized by the one-loop corrections of the preceding section.From Eq.4.10) we find

$$
\Omega_{i k}\left(\mu_{x}, s\right)=\Omega^{(1)}\left(\mu_{x}, s\right) \delta_{i k}\left(1+\delta_{i 3}\right),
$$

and

$$
\boldsymbol{R}\left(\mu_{x}, s\right)=\frac{1}{2} \frac{d \omega_{s}^{(1)}\left(\mu_{x}, s\right)}{d \mu_{x}}(\boldsymbol{F} \times \boldsymbol{N}) .
$$

The spin current tensor takes the form 


$$
J_{k i}(\boldsymbol{x} \mid E, s)=-D_{s}^{(1)}\left(\mu_{x}, s\right) \nabla_{k} S_{i}(\boldsymbol{x} \mid E, s)+\omega_{s}^{(1)}\left(\mu_{x}, s\right)\left(N_{i} S_{k}(\boldsymbol{x} \mid E, s)-\delta_{i k}(\boldsymbol{N} \cdot \boldsymbol{S}(\boldsymbol{x} \mid E, s))\right) .
$$

This tensor has been obtained by collecting all those terms in Eq. 4.10), which can be cast into a divergence. Accordingly, the tensor (5.4) might deviate from the true spin-current tensor by terms which are annihilated by the divergence. However, since every observable quantity can be calculated directly from Eq.(5.1) such terms are not important for our further considerations.

The Eqs.4.14)-4.22) ignore the impact of inelastic scattering events on the transport coefficients. To take them into account we introduce the phenomenological phase relaxation time $\tau_{\phi}(T)$ and replace $s \rightarrow s+1 / \tau_{\phi}(T)$. In this case the transport coefficients are given by the Eqs.(5.2) and (5.3) with $s$ replaced by $1 / \tau_{\phi}(T)$ for $s \ll$ $1 / \tau_{\phi}(T)$. Accordingly, the relaxation becomes Markovian for $t>\tau_{\phi}(T)$. Below we restrict the consideration to this situation.

\section{A. Decay of a homogeneous initial magnetization}

In order to investigate some of the consequences of Eq. (5.1) we first focus on the decay of a spatially homogeneous magnetization. To this end we assume that an initial magnetization $\boldsymbol{S}_{0}$ has been created at time $t=0$ and that only charge carriers in the vicinity of the Fermienergy $\mu$ contribute to the magnetization. In this case the initial distribution function is given by

$$
\left.\boldsymbol{S}^{0}\right|_{F=0}(\boldsymbol{x}, E)=\boldsymbol{S}_{0} \delta(\mu-E),
$$

in the absence of the field. In the presence of the field the energy of a charge carrier placed at $\boldsymbol{x}$ is changed by $\boldsymbol{F} \cdot \boldsymbol{x}$. Therefore, the initial condition takes the form

$$
\begin{aligned}
\boldsymbol{S}^{0}(\boldsymbol{x}, E) & =\boldsymbol{S}_{0} \delta(\mu+\boldsymbol{F} \cdot \boldsymbol{x}-E) \\
& =\boldsymbol{S}_{0} \delta\left(\mu-\mu_{x}\right)
\end{aligned}
$$

in the presence of the field.

During the evolution the spin distribution remains spatially homogeneous. Accordingly, $\boldsymbol{S}(\boldsymbol{x} \mid E, s)=\boldsymbol{S}\left(\mu_{x}, s\right)$. The direction and the magnitude of the magnetization and the energy of the particles are changed. Since, however, the magnetization decays on a time scale small compared to the time needed by a particle to change its energy appreciably the energy distribution function remains close to the initial delta-function during the time in which the magnetization is measurable. To take advantage of this fact we make the ansatz

$$
\boldsymbol{S}\left(\mu_{x}, s\right)=\boldsymbol{S}(s) \delta\left(\mu-\mu_{x}\right)
$$

and derive a closed equation for $\boldsymbol{S}(s)$ by integrating Eq. (5.1) with respect to $\mu_{x}$. Doing so, we obtain the simple

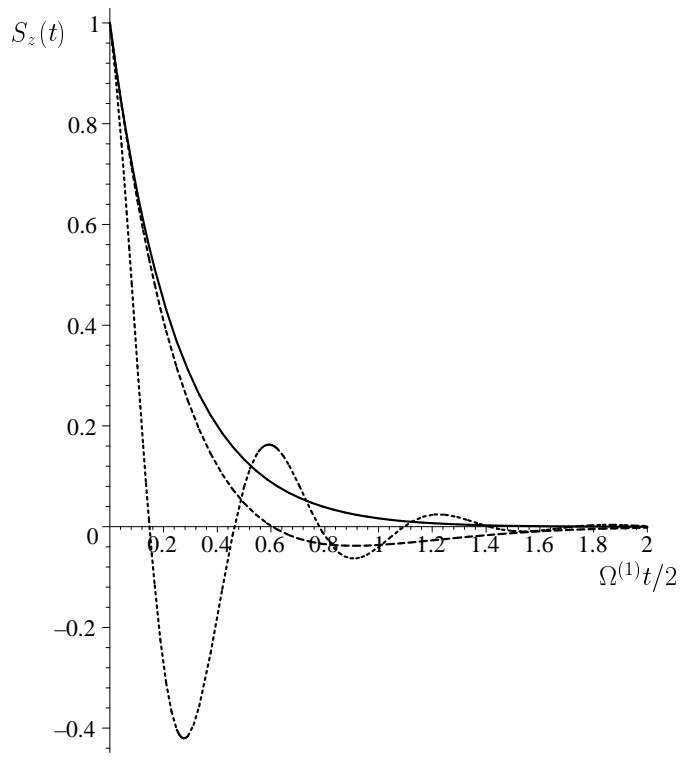

FIG. 1: Decay of $S_{z}$ for $2 \Omega_{F} / \Omega^{(1)}=0.3$ (solid line), 2 (dashed line) and 10 (dashed dotted line). The initial conditions are $S_{z}(0)=1$ and $S_{x}(0)=0$.

algebraic system of equations

$$
\left(s+\Omega^{(1)}(\mu)\right) S_{x}(s)-\frac{1}{2} \frac{d \omega_{s}^{(1)}(\mu)}{d \mu}[(\boldsymbol{N} \times \boldsymbol{F}) \times \boldsymbol{S}(s)]_{x / y}=S_{x / y}^{0},
$$

$$
\left(s+2 \Omega^{(1)}(\mu)\right) S_{z}(s)-\frac{1}{2} \frac{d \omega_{s}^{(1)}(\mu)}{d \mu}[(\boldsymbol{N} \times \boldsymbol{F}) \times \boldsymbol{S}(s)]_{z}=S_{z}^{0} .
$$

This system has the same structure as that for the decay of an initial magnetization in an ordered system (hopping transport of small polarons on an ordered lattice $)^{22}$. Only the structure of the transport coefficients is different.

To calculate the magnetization we solve this system and perform an inverse Laplace transformation. Doing so, we obtain

$$
S_{y}(t)=S_{y}^{0} e^{-\Omega^{(1)} t},
$$

$$
\begin{aligned}
S_{x}(t)=e^{-3 \Omega^{(1)} t / 2}[ & S_{x}^{0}\left[\cosh \left(\omega_{F} t\right)+\frac{\Omega^{(1)}}{2} \frac{\sinh \left(\omega_{F} t\right)}{\omega_{F}}\right] \\
& \left.+S_{z}^{0} \Omega_{F} \frac{\sinh \left(\omega_{F} t\right)}{\omega_{F}}\right]
\end{aligned}
$$




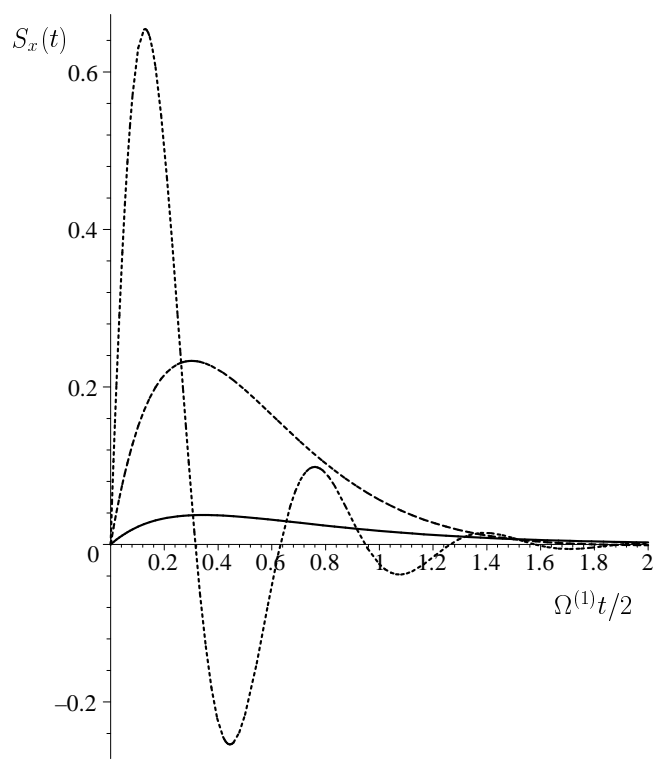

FIG. 2: $S_{x}(t)$ for $S_{z}(0)=1$ and $S_{x}(0)=0$. The parameters are the same as in Fig.1.

and

$$
\begin{aligned}
S_{z}(t)=e^{-3 \Omega^{(1)} t / 2}[ & S_{z}^{0}\left[\cosh \left(\omega_{F} t\right)-\frac{\Omega^{(1)}}{2} \frac{\sinh \left(\omega_{F} t\right)}{\omega_{F}}\right] \\
& \left.-S_{x}^{0} \Omega_{F} \frac{\sinh \left(\omega_{F} t\right)}{\omega_{F}}\right] .
\end{aligned}
$$

Here $\Omega^{(1)} \equiv \Omega^{(1)}(\mu)$,

$$
\omega_{F}=\sqrt{\Omega^{(1)^{2}}-4 \Omega_{F}^{2}} / 2
$$

and

$$
\Omega_{F}=\frac{1}{2} \frac{d \omega_{s}(\mu)}{d \mu} F N_{z}
$$

The Eqs.(5.10)-(5.12) apply for $t>\tau_{\phi}(T)$. They do not contain the magneto-electric effect of Ref. [23], since this effect is proportional to the ratio between the Rashba level splitting and the Fermi energy, which is considered as small in our treatment.

According to the Eqs. (5.10)-(5.14) the character of the decay of the initial magnetization depends on the magnitude of the electric field. If $F<F_{c}$, where $F_{c}$ is determined from the requirement $\Omega^{(1)}=2\left|\Omega_{F_{c}}\right|$, the frequency $\omega_{F}$ is real and reflects itself in an exponential decay of an initial magnetization (see the Figs.1 and 2). Furthermore, the $x$-component of the magnetization is coupled with the $z$-component of the magnetization. If $S_{z}^{0} \neq 0$ a non vanishing magnetization in $x$-direction is created even if $S_{x}^{0}=0$. If $F>F_{c}$ the frequency $\omega_{F}$ becomes purely imaginary. In this case the hyperbolic functions turn to trigonometric functions, which lead to a rotation of the decaying initial magnetization (see Fig.1 and 2).
Therefore, the vector $\left(S_{x}(t), S_{z}(t)\right)$, with components

$$
\begin{aligned}
S_{x}(t)=e^{-3 \Omega^{(1)} t / 2}[ & S_{x}^{0}\left[\cos \left(\left|\omega_{F}\right| t\right)+\frac{\Omega^{(1)}}{2} \frac{\sin \left(\left|\omega_{F}\right| t\right)}{\left|\omega_{F}\right|}\right] \\
& \left.+S_{z}^{0} \Omega_{F} \frac{\sin \left(\left|\omega_{F}\right| t\right)}{\left|\omega_{F}\right|}\right]
\end{aligned}
$$

and

$$
\begin{aligned}
S_{z}(t)=e^{-3 \Omega^{(1)} t / 2}[ & S_{z}^{0}\left[\cos \left(\left|\omega_{F}\right| t\right)-\frac{\Omega^{(1)}}{2} \frac{\sin \left(\left|\omega_{F}\right| t\right)}{\left|\omega_{F}\right|}\right] \\
& \left.-S_{x}^{0} \Omega_{F} \frac{\sin \left(\left|\omega_{F}\right| t\right)}{\left|\omega_{F}\right|}\right],
\end{aligned}
$$

moves on an ellipse with decaying diameter. The ellipse satisfies the equation

$\Omega_{F} S_{x}^{2}(t)+\Omega S_{x}(t) S_{z}(t)+\Omega_{F} S_{z}^{2}(t)=$ const $\cdot \exp \left(-3 \Omega^{(1)} t\right)$

The quadratic form can be diagonalized. Doing so, it can be shown that the elliptic axis are tilted by $\pi / 4$.

\section{B. Alternating Hall-current}

The rotation of the magnetization discussed in the preceding subsection reflects also in the current. In the presence of the Rashba interaction the current operator has the form 24.25

$$
\hat{\boldsymbol{j}}(t)=e \frac{\hat{\boldsymbol{p}}(t)}{m}-\frac{2 e}{\hbar} \hat{\boldsymbol{S}}(t) \times \boldsymbol{N} .
$$

Here $e$ is the charge and $\hat{\boldsymbol{S}}$ is the spin operator. The Heisenberg equation for $\hat{\boldsymbol{p}}(t)$ is independent of $\hat{\boldsymbol{S}}(t)$. Accordingly, the configuration average of the expection value of the first term on the right hand side of Eq.(5.18) yields the conventional Drude current and its weak localization corrections. The configuration average of the second term yields two additional contributions, a correction term to the longitudinal current

$$
\delta j_{x}(t)=-\frac{2 e}{\hbar} N_{z} S_{y}(t),
$$

and the Hall-like term

$$
j_{y}(t)=\frac{2 e}{\hbar} N_{z} S_{z}(t)
$$

perpendicular to both $\boldsymbol{F}$ and $\boldsymbol{N}$. If we chose the same initial condition for the magnetization as in the preceding section $S_{z}(t)$ is oscillating, if $2\left|\Omega_{F}\right|>\Omega^{(1)}$. According to Eq.(5.20) these oscillations manifest themselves in an alternating Hall-like current.The alternating Hall current is produced by a constant electric field. However, since the magnetization decays when time elapses the current oscillations are not stable but decay too. This raises the interesting question whether these oscillations can be stabilized by some means. 


\section{RESULTS}

In the paper we have used field theoretical methods to derive the generalized Bloch equations for the magnetization in a disordered two-dimensional electron gas. Our equations apply for time scales which are large compared to the elastic scattering time, large compared to the phase-breaking time but small compared to energy relaxation time. Accordingly, they take into account the particle- and spin diffusion, the drift induced by an external electric field in the plane, the heating of the charge carriers, the decay of the magnetization due to scattering events, and an electric field dependent rotation of the magnetization direction.

The calculation of the transport coefficients shows that the impact of the quantum-corrections on the spin transport coefficients is different from that on the particle transport coefficients. The corrections to the latter are antilocalizing. This fact manifests itself in a minimum of the diffusion coefficient as a function of the temperature. The structure of the corrections to the particle transport coefficients agrees with the conventional antilocalization corrections to the conductivity due to spin-orbit coupling $^{21}$. In contrast to the particle transport coefficients the spin transport coefficients show a tendency to localization. Both the spin diffusion coefficient and the spin decay rate decrease with decreasing temperature, even at very low temperatures. This sets the situation for the spin-transport coefficients apart from that for the particle transport coefficients, which increase with decreasing temperature at sufficiently low temperature due to antilocalization.

We have applied these equations to an investigation of the decay of a spatially homogeneous initial magnetization. In the absence of the in-plane electric field such a magnetization decays exponentially at large $\operatorname{times}^{26}$. The decay is anisotropic. Its transverse component decays twice as fast as its longitudinal components. The temperature dependence of the decay is given by the temperature dependence of the weak localization corrections to the transport coefficients, which lead to a logarithmic reduction of the decay rate with decreasing temperature. Accordingly, the decay becomes slower with decreasing temperature.

Already a weak electric field renders the decay qualitatively different. If the electric field is smaller than a critical field it tips the magnetization only slightly over. If, however, the field exceeds the critical field $F_{c}$ the magnetization starts rotating in the plane transverse to $\boldsymbol{N} \times \boldsymbol{F}$. The rotation frequency depends on the degree of disorder, on the magnitude of the Rashba-parameter, on the magnitude of the electric field and on temperature. An increase of the temperature results also in an increase of the rotation frequency $\omega_{F}$ and in an increase of the critical field $F_{c}$. We would like to note that a similar rotation has also been obtained in Ref. 22] for small polarons on an ordered lattice. Our calculation shows that this rotation can also be observed in a disordered electron gas, in which the transport mechanism is very different from that for a small polaron on an ordered lattice.

\section{Acknowledgments}

This work was done during a stay at the the University of Oregon. The author is grateful for the hospitality of the material science institute, in particular to D. Belitz, without whose support and encouragement this work would not have been accomplished. I would also like to thank V. V. Bryksin for helpful discussions. This work was supported by the DFG under grant. No. Bl456/3-1.
1 S. Datta and B. Das, Appl. Phys. Lett. 56, 665 (1990).

${ }^{2}$ R. Fiederling, M. Keim, G. Reuscher, W. Ossau, G. Schmidt, A. Waag, and L. W. Molenkamp, Nature (London) 402, 787 (1999).

3 Y. Ohno, D. K. Young, B. Beschoten, F. Matsukura, H. Ohno and D. D. Awschalom, Nature (London) 402, 790 (1999).

4 H. J. Zhu, M. Ramsteiner, H. Kostial, M. Wassermeier, H. P.Schonherr and K. H. Ploog, Phys. Rev. Lett. 87, 016601 (2001).

5 G. Schmidt, G. Richter, P. Grabs, C. Gould, D. Ferrand and L. W.Molenkamp, Phys. Rev. Lett. 87, 227203 (2001).

6 P. R. Hammar and M. Johnson, Phys. Rev. Lett. 88, 066806 (2002).

7 Jun-ichiro Inoue, G. E. W. Bauer and L. W. Molenkamp, Phys. Rev. B 67, 33104 (2003).

8 S. D. Ganichev, E. L. Ivchenko, V. V. Bel'kov, S. A. Tarasenko, M. Sollinger, D. Weiss, W. Wegschneider and W. Prettl, Nature (London) 417, 153 (2002).

9 J. Sinova, D. Culcer, Q. Niu, N. A. Sinitsyn, T. Jungwirth, and A. H. MacDonald, cond-mat 0307663

10 I. L. Aleiner and V.I. Fal'ko, Phys. Rev. Lett. 87, 256801 (2001).

11 P. W. Brouwer, J. N. H.J. Cremers, and B. I. Halperin, Phys.Rev.B65, 081302(R) (2002).

12 D. M. Zumbühl, J. B. Miller, C. M. Marcus, K. Campman, and A. C. Gossard, Phys. Rev. Lett. 89, 276803 (2002).

13 Takaaki Koga, Junsaku Nitta, Tatsushi Akazaki, and Hideaki Takayanagi, Phys. Rev. Lett. 89, 46801 (2002).

14 J. B. Miller, D. M. Zumbühl, C. M. Marcus, Y. B. LyandaGeller, D. Goldhaber-Gordon, K. Campman, and A. C. Gossard, Phys. Rev.Lett.90, 76807 (2003).

15 J. Schliemann, J. C. Egues and D. Loss, Phys. Rev. Lett. 90, 146801 (2003).

16 A. J. McKane and M. Stone, Ann. Phys. (NY) 131, 36 (1981).

17 A. M. M. Pruisken and L. Schäfer, Nucl. Phys. B 200 [FS4], 20 (1982).

18 D. Belitz and T. R. Kirkpatrick, Rev. Mod. Phys. 66, 261 
(1994).

19 L. Kadanoff and G. Baym, Quantum Statistical Mechanics, Addison-Wesley Pub. Comp.

${ }^{20}$ O. Bleibaum and D. Belitz, submitted to Phys. Rev. B. (cond-mat/0309470).

21 S. Hikami, A. I. Larkin and Y. Nagaoka, Prog. Theor. Phys. 63, 707 (1980).

22 T. Damker, H. Böttger and V. V. Bryksin, phys. stat. sol.(b) in print.
${ }^{23}$ V. M. Edelstein, Solid State. Commun. 73, 233 (1990).

24 L. W. Molenkamp, G. Schmidt and G. E. W. Bauer, Phys. Rev. B 64, 121202(R) (2001).

25 U. Zülicke and C. Schroll, Phys. Rev. Lett. 88, 29701 (2002).

26 The magneto-electric effect of Ref. 23] is absent in our approach since we have ignored the Rashba level splitting compared to the Fermi energy. 